\title{
Bajo desempeño escolar relacionado con la persistencia del tabaquismo en una cohorte de estudiantes en México
}

\author{
Rafael Anaya-Ocampo, M en C,(1) Edna Arillo-Santillán, M en C,(1) \\ Luisa María Sánchez-Zamorano, Dr en C, (1) Eduardo Lazcano-Ponce, Dr en C.(1)
}

\begin{abstract}
Anaya-Ocampo R, Arillo-Santillán E, Sánchez-Zamorano LM, Lazcano-Ponce E. Bajo desempeño escolar relacionado con la persistencia del tabaquismo en una cohorte de estudiantes en México. Salud Publica Mex 2006;48 supl I:SI7-S29.
\end{abstract}

\section{Resumen}

Objetivo.Evaluar el efecto independiente de la persistencia del tabaquismo en el bajo desempeño escolar en un estudio poblacional prospectivo; para ello se utilizaron dos mediciones en un periodo de tres años aplicadas a sujetos que permanecieron en el ámbito escolar durante el lapso de seguimiento. Material y métodos. Se desarrolló un estudio de cohorte en el ámbito escolar en los periodos 19981999 y 2000-200I. En este artículo sólo se incluyó a 2568 estudiantes (persistentes en el tabaquismo y no consumidores). Se construyeron modelos de regresión logística múltiple a partir de dos estratos: estudiantes con mantenimiento o mejoría del desempeño escolar e individuos con bajo rendimiento. Se consideraron dos mediciones. Se evaluó la diferencia de riesgos de persistencia del tabaquismo en función del desempeño escolar y covariables relacionadas.Resultados. En la cohorte de referencia, 5.2\% de los estudiantes continuó el consumo de tabaco durante el periodo de estudio, mientras que $64.2 \%$ permaneció al margen de la exposición. El bajo desempeño escolar se vinculó con la persistencia del tabaquismo en la población total $(R M=2.27 ;$ IC95\% I.4-3.6), así como en mujeres (RM= $3.0 ;$ IC95\% I.4-6.7) y hombres ( $R M=2.2$; IC95\% I.I-4.I). El bajo rendimiento escolar tiene un exceso de riesgo de continuación del tabaquismo, independiente de otras covariables. La mayor diferencia en términos de prevalencia, respecto del bajo desempeño escolar, se reconoció en los sujetos con síntomas depresivos con un exceso de persistencia del tabaquismo (21.3\%). Conclusiones. Deben plantearse en forma integral intervenciones que fomenten estilos
Anaya-Ocampo R, Arillo-Santillán E, Sánchez-Zamorano LM, Lazcano-Ponce E. Poor school perfomance associated with tobacco persistence among Mexican students. Salud Publica Mex 2006;48 suppl I:S 17-S29.

\begin{abstract}
Objective. To evaluate the independent effect of poor school performance and tobacco persistence using a prospective population study of students who remained on school grounds during the follow-up period. Two measurements were taken over a period of three years. Material and methods. A cohort study conducted within school confines was developed over the period 1998 to 1999 and 2000 to 200 I, with 2568 students, including both tobacco persistent students and non-consumers. Multiple logistic regression models were designed and structured according to educational performance using two standards: students who maintained and/or improved performance and those with poor performance, based on the first and second measurements. Results. In the reference cohort, $5.2 \%$ of the students reported to have persisted with tobacco consumption during the study period, while $64.2 \%$ remained unexposed. Poor school performance was associated with tobacco persistence for the total population (OR 2.27,95\% CI I.4-3.6), as well as for females (OR $3.095 \% \mathrm{Cl}$ I.4-6.7) and males (OR $2.295 \% \mathrm{Cl}$ I.I-4.I). Poor school performance has an excess risk of tobacco persistence, independently of other co-variables. The greatest difference in prevalence, using poor school performance as a reference, was observed in those subjects having depressive symptomolgy, with an excessive tobacco persistence of $21.3 \%$. Conclusions. Interventions that foster healthy lifestyles within school environments should be adopted in a comprehensive manner. In addition, students who perform poorly should be considered vulnerable to unhealthy lifestyles. It will therefore be necessary to
\end{abstract}

(I) Centro de Investigación en Salud Poblacional, Instituto Nacional de Salud Pública. Cuernavaca, Morelos, México

Fecha de recibido: 7 de marzo de 2006 - Fecha de aprobado: 2 I de abril de 2006 Solicitud de sobretiros: Dr. Eduardo Lazcano Ponce. Director del Centro de Investigación en Salud Poblacional, Instituto Nacional de Salud Pública. Av. Universidad 655, Col. Sta. María Ahuacatitlán, 62508 Cuernavaca, Morelos, México.

Correo eléctrónico: elazcano@correo.insp.mx 
de vida saludables en el ámbito escolar. Asimismo, los estudiantes con bajo rendimiento escolar deben considerarse sujetos vulnerables a estilos de vida no saludables, por lo que es necesario identificarlos como individuos de alto riesgo e instituir intervenciones en este grupo poblacional, en particular en el combate a las adicciones.

Palabras clave: desempeño escolar; tabaquismo; persistencia; cohorte; adolescentes; México identify them as high risk subjects and implement interventions among this population, particularly with regard to combatting addictions.

Keywords: school performance; tobacco; persistence; adolescents; Mexico
$\mathrm{L}$ a escuela constituye uno de los ámbitos relevantes donde se lleva a cabo la socialización y el desarrollo de los adolescentes; otros son la familia y el grupo de pares. El ámbito escolar es un nicho de oportunidad para desarrollar conductas saludables, pero, a su vez, la inadaptación escolar puede influir en comportamientos de riesgo, entre los que figuran el consumo inmoderado de alcohol, tabaco y drogas ilegales o las prácticas sexuales de alto riesgo, además de conductas antisociales y alteraciones del comportamiento. ${ }^{1}$ En general, existe evidencia científica que muestra una relación entre el estilo de vida saludable, una mejor relación con el contexto escolar y un desempeño escolar exitoso y satisfacción escolar consecuentes, así como la intención de proseguir el estudio. ${ }^{1}$

La prevalencia del consumo de tabaco entre los adolescentes se ha estudiado sobre todo a través de conductas contaminantes, que definen el comportamiento humano como el resultado de creencias, actitudes e intenciones, social e históricamente determinadas. ${ }^{2} \mathrm{El}$ término persistencia se define como "la acción de mantenerse firme o constante en un hábito o la constancia en el intento de alguna actividad"; 3 en el análisis epidemiológico se utiliza para referirse al uso continuo de sustancias adictivas ${ }^{4,5}$ y la presencia de conductas de riesgo que pueden vincularse con el incremento de enfermedades o la mortalidad misma. ${ }^{6}$

Respecto de los logros académicos, algunos trabajos refieren que el uso y la experimentación del tabaco entre los adolescentes se relacionan de modo negativo con los logros académicos, ${ }^{7,8}$ medidos, entre otros elementos por el desempeño escolar y, a su vez, con una gran variedad de factores psicológicos, sociales, económicos y personales del adolescente. ${ }^{9,10}$ En términos conceptuales, el desempeño escolar se define como el nivel de conocimientos demostrado en un área, en comparación con la norma de edad y nivel académico. ${ }^{11} \mathrm{Su}$ evaluación implica diversos aspectos individuales y sociales que afectan de manera positiva o negativa la vida académica de los estudiantes. Los aspectos como el ambien- te escolar, la metodología didáctica de los profesores y las prácticas que llevan al fortalecimiento del conocimiento determinan en gran medida la adquisición de las distintas habilidades académicas. Para este estudio se consideró que el autoinforme de calificaciones escolares refleja el rendimiento académico del individuo entrevistado; se asume que el desempeño académico es un fenómeno multifactorial, con predictores psicológicos y determinantes sociales, entre los que se encuentran la edad, la autoestima, el nivel socioeconómico y el lugar de residencia. ${ }^{4,12}$ Notificaciones previas han señalado que el bajo rendimiento académico se relaciona con problemas adictivos y está influido -entre otros factores- por las relaciones familiares, la presencia de adicciones en los padres y las interacciones con los amigos y conocidos (influencia de pares). ${ }^{4,9,13,14}$ En resumen, la persistencia en el consumo de tabaco es consecuencia de un estilo de vida, en el marco de ciertas condiciones de vida que afecta en grado negativo el desempeño escolar y, bajo ciertas circunstancias, reduce la posibilidad de alcanzar logros académicos.

Un estudio anterior en adolescentes mexicanos mostró el posible nexo entre rendimiento escolar y consumo de tabaco, alcohol y drogas ilegales. ${ }^{8}$ En esta cohorte de estudiantes del estado de Morelos se analiza una vinculación inversamente proporcional, que revela que un adolescente con bajo desempeño escolar tiene más riesgo de ser susceptible a la presencia y persistencia del consumo de tabaco, al margen de otros factores. Además, la continuación en el tabaquismo en adolescentes es un sólido predictor para determinar dicha adicción en su futura vida adulta. ${ }^{15}$

\section{Material y métodos}

\section{Diseño del estudio}

La información proviene de una investigación multidisciplinaria e interinstitucional que realizó en el estado de Morelos el Instituto Nacional de Salud Pública 
(INSP), en colaboración con las Secretarías de Salud y Educación Pública locales, instituciones educativas y organizaciones no gubernamentales. Se trata de un estudio de cohorte efectuado en el ámbito escolar de los niveles secundaria, bachillerato y licenciatura, con representación estatal, entre los años de 1998 y 1999, periodo en el que se llevó a cabo la medición basal, y 2000 y 2001, para la segunda medición, en la que participaron estudiantes adolescentes y adultos jóvenes.

En la medición basal, la muestra incluyó a escuelas de los 33 municipios y 72 distritos escolares de Morelos. Mediante un proceso de selección aleatoria se identificó a 94 escuelas; éstas forman parte de los niveles de educación secundaria, preparatoria o bachillerato y nivel superior. ${ }^{16}$ Se obtuvo un total de 13293 sujetos de estudio entre 11 y 24 años de edad. Para la segunda medición, con base en la factibilidad del seguimiento de aquellos individuos que continuaron en el sistema público de enseñanza del estado (figura 1), se realizó una fracción de muestreo por grupos de edad en 33\% de la muestra original y se obtuvo una tasa de respuesta de $94.8 \%$. La deserción escolar en el periodo de estudio es muy alta, cercana a 30\%. La búsqueda de los elegidos se realizó en sus domicilios y en los niveles de enseñanza posteriores a la primera ocasión, en particular en los niveles bachilerato y profesional. Hasta $90 \%$ de las entrevistas se efectuó en el interior de los planteles escolares y $10 \%$ en los domicilios, de acuerdo con la época del año en que se llevó a cabo la encuesta. En esta fase se efectuó la segunda entrevista y el estudio correspondiente de un total de 3699 individuos, a quienes se aplicaron los instrumentos de recolección de información utilizados en la medición basal. Para fines de este estudio, se empleó una submuestra de población de 2568 individuos, quienes integran una cohorte de estudiantes persistentes en el tabaquismo y no consumidores.

La variable dependiente de persistencia en el consumo de tabaco se construyó a partir de las respuestas del estudiante que en ambas mediciones respondió positivamente a la pregunta: ¿tú fumas cigarrillos?, y además fumaba al menos un cigarrillo al día (fumador actual). La población de comparación la conformaron los estudiantes que respondieron "no" a la misma pregunta y se consideró a éstos como no fumadores o persistentes en el "no consumo de tabaco". Se excluyó de este análisis a los estudiantes que cambiaron su condición de una medición a otra: quienes señalaron que iniciaron el hábito y los que dejaron de fumar, o experimenta-

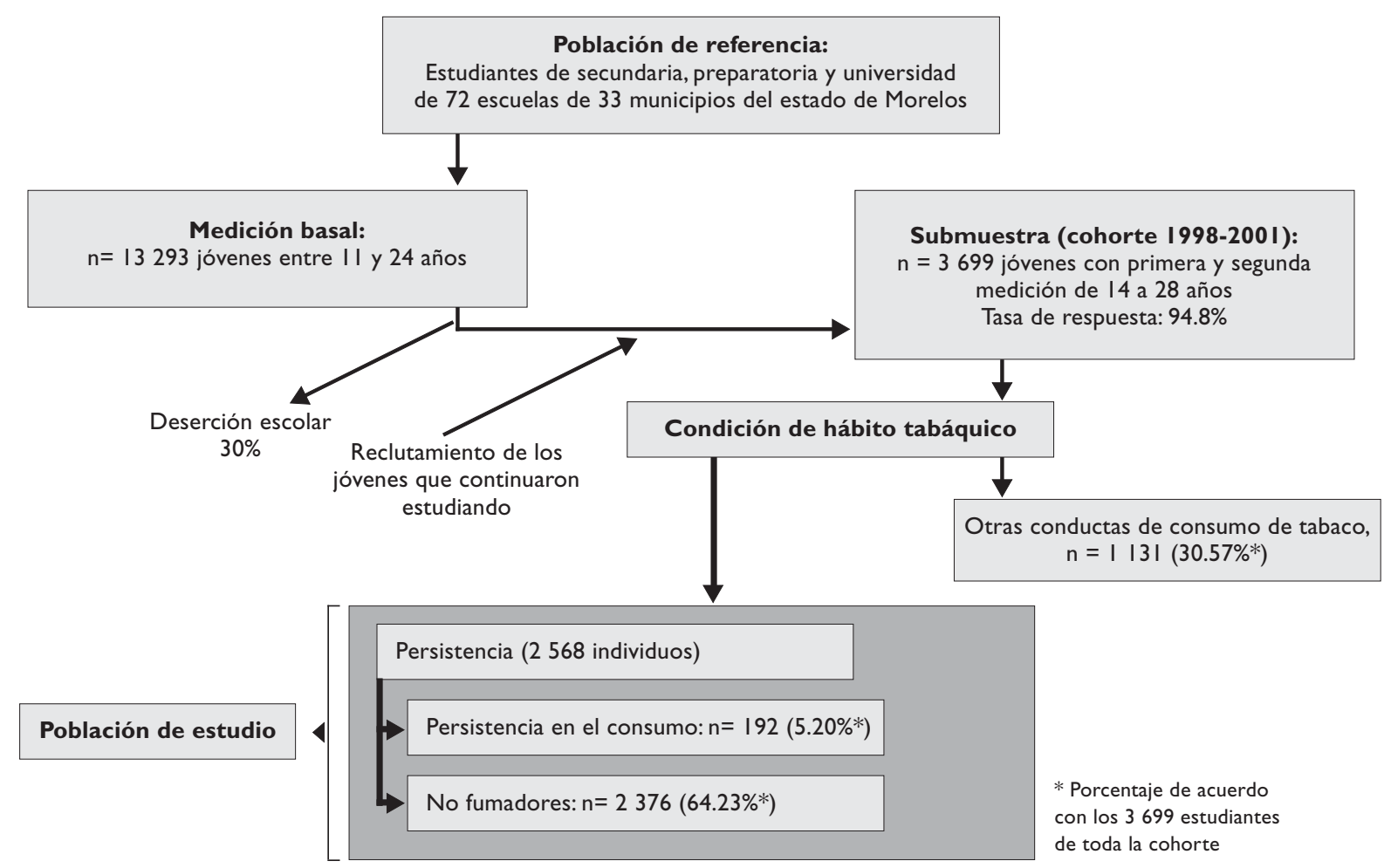

Figura I. Muestreo de jóvenes que continuaron en la educación pública en relación con el tabaquismo. Morelos, México 
dores. Así, se obtuvo una muestra de 2568 individuos persistentes o apartados del hábito de fumar.

La variable independiente principal es el desempeño escolar, medido a través del autoinforme del promedio de calificaciones obtenido en el último año escolar. Esta información procedió de la medición basal y de la segunda medición. En cada una de éstas, la variable discreta del promedio de calificaciones se agrupó en dos categorías: a) promedio $\leq 7 \mathrm{y}$ b) promedio $\geq 8$. La combinación de ambas mediciones resultó en cuatro categorías: a) aquellos que mantuvieron un promedio $\geq 8$ en ambas mediciones, $b$ ) los que subieron de promedio, c) quienes bajaron de promedio; $\mathrm{y} d$ ) aquellos que mantuvieron un promedio $\leq 7$ en ambas mediciones. Después se recategorizó con valores de 0 y 1 ; la categoría 0 incluye a quienes mantuvieron un promedio $\geq 8$ en ambas mediciones y los que mejoraron dicho promedio; la categoría 1 la constituyeron quienes conservaron un promedio $\leq 7$ en las dos mediciones y aquellos que bajaron de promedio.

Otras covariables fueron el sexo, la edad, el nivel socioeconómico, el lugar de residencia, el consumo inmoderado de alcohol, el consumo de drogas ilegales, el antecedente de relaciones sexuales, la percepción de tabaquismo en los padres, el consumo de drogas en amigos y los síntomas vinculados con depresión. En la variable sexo se consideró como referencia a las mujeres. Para la variable cohorte de nacimiento se construyeron cuatro categorías de acuerdo con la edad notificada en la segunda medición $(0=14$ a 16 años; $1=17$ a $19 ; 2=20$ a 22; y $3=23$ o más años); las variables de nivel socioeconómico y residencia se dividieron en tres categorías ( $0=$ bajo; $1=$ medio; y 2 = alto; y $0=$ rural; $1=$ semiur bano; y 2 = urbano, respectivamente) y se computaron con los datos de la primera medición que se consideraron variables fijas en el tiempo; la variable intoxicación por alcohol se dividió en tres categorías (nunca, ocasionalmente y frecuentemente); el consumo de drogas se construyó como sigue: antecedentes de consumo de alguna droga ilegal en la vida (cocaína, marihuana, heroína, entre otras) o sin ellos. En la variable de relaciones sexuales se consideró a quienes las iniciaron en comparación con aquellos que respondieron de forma negativa en ambas mediciones. El tabaquismo en padres se planteó en términos de la persistencia de que al menos un padre fuera consumidor o la ausencia de padres consumidores, información señalada de acuerdo con la percepción del estudiante. Respecto de la variable amigos que consumen drogas, se consideró el antecedente de convivir con amigos consumidores, en comparación con quienes indicaron no tener amigos consumidores. Para los síntomas relacionados con la depresión, se usó el primer factor de la escala de Zung y se consideraron los datos de la medición basal.

\section{Análisis de la información}

La descripción de la población de estudio se estratificó por sexo, debido a que los patrones de consumo de tabaco en hombres y mujeres eran diferentes. ${ }^{17}$ Se realizó un análisis descriptivo de la base de datos integrada por ambas mediciones, para comparar los cambios porcentuales a lo largo del tiempo de las variables incluidas en el estudio; por tal razón, se obtuvo el cálculo de la diferencia de proporciones, mediante la prueba exacta de Fisher entre las mediciones basal y segunda, y se estratificó por sexo para cada una de las variables del estudio. Se obtuvieron además las prevalencias de persistencia en el consumo de tabaco para cada una de las variables incluidas en el estudio, estratificadas por desempeño escolar, y se computó el exceso de riesgo de la persistencia de tabaquismo en función del rendimiento escolar.

Al considerar una posible modificación de efecto por sexo, se desarrolló un modelo de regresión múltiple que incluyó términos de interacción entre esta variable y cada una de las variables del estudio. Con base en los resultados obtenidos de este modelo, se construyó otro modelo de regresión logística múltiple para toda la población, que incluyera los términos de interacción que resultaron significativos, además de ajustar las potenciales variables de confusión consideradas en el estudio.

Por otra parte, a través de modelos de regresión logística múltiple estratificados por desempeño escolar, se buscó identificar las variables relacionadas con mantener alto o mejorar el rendimiento y las variables vinculadas con mantener bajo o empeorar el desempeño escolar. En los modelos de regresión logística múltiple se registraron las razones de momios $(R M)$ con un intervalo de confianza de 95\% (IC95\%) y el valor de significancia $(p<0.05)$. Para el análisis de los datos se utilizó el paquete estadístico STATA versión 8.2.*

\section{Resultados}

La población total de la cohorte de referencia se integró con 3699 estudiantes (en el cuadro I se muestra la distribución de las características estudiadas). En relación con el desempeño escolar, esta variable de exposición fue estable a lo largo del tiempo, de tal manera que sólo $4.2 \%$ de las mujeres redujo su rendimiento escolar en el periodo de estudio (una observación similar se advierte en hombres con $4.8 \%$ ). En las 2244

\footnotetext{
* Stata Corp. Stata Statistical Software: Release 8.2 Collage Station. Texas: Stata Corporation, 2003.
} 


\section{Cuadro I \\ DifERENCIAS DE LA DISTRIBUCIÓN DE LAS CARACTERÍSTICAS DE LA POBLACIÓN TOTAL ( $\mathrm{N}=3$ 699) DE LA COHORTE DE ACUERDO A LA MEDICIÓN BASAL Y SEGUNDA MEDICIÓN, ESTRATIFICADA POR SEXO en estudiantes de Morelos, 1998-200I.}

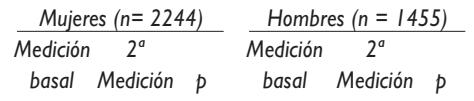

Desempeño escolar

\begin{tabular}{lllllll} 
Calificación $\geq 8$ & 86.1 & 81.9 & 0.00 & 77.2 & 72.4 & 0.00 \\
\hline Calificación $\leq 7$ & 13.9 & 18.1 & 0.00 & 22.8 & 27.6 & 0.00
\end{tabular}

Lugar de residencia

\begin{tabular}{lllllll} 
Rural & 38.3 & 39.2 & 0.51 & 44.5 & 34.6 & 0.00 \\
\hline Semiurbano & 25.8 & 21.8 & 0.00 & 21.5 & 16.1 & 0.00 \\
\hline Urbano & 35.9 & 39.0 & 0.03 & 34.0 & 49.3 & 0.00
\end{tabular}

Consumo de tabaco

\begin{tabular}{lrrrrrr} 
No & 92.0 & 85.6 & 0.00 & 84.5 & 73.0 & 0.00 \\
\hline Sí & 8.0 & 14.4 & 0.00 & 15.5 & 27.0 & 0.00
\end{tabular}

Intoxicación aguda por alcohol

\begin{tabular}{lrrrrrr} 
Nunca* & 79.5 & 62.4 & 0.00 & 73.7 & 52.2 & 0.00 \\
\hline Ocasionalmente $^{\ddagger}$ & 19.7 & 35.5 & 0.00 & 24.1 & 41.6 & 0.00 \\
\hline Frecuentemente & 0.8 & 2.1 & 0.00 & 2.2 & 6.2 & 0.00
\end{tabular}

Historia de consumo de drogas ilegales

\begin{tabular}{lrrrrrr} 
No & 98.8 & 97.5 & 0.00 & 94.9 & 92.4 & 0.00 \\
\hline Sí & 1.2 & 2.5 & 0.00 & 5.1 & 7.6 & 0.00
\end{tabular}

Antecedente de relaciones sexuales

\begin{tabular}{lrrrrrr} 
No & 94.2 & 82.3 & 0.00 & 85.4 & 69.6 & 0.00 \\
\hline Sí & 5.8 & 17.7 & 0.00 & 14.6 & 30.4 & 0.00
\end{tabular}

Percepción de tabaquismo en padres

\begin{tabular}{lrrrrrr} 
Ninguno $^{\S}$ & 78.3 & 64.8 & 0.00 & 77.7 & 63.9 & 0.00 \\
\hline En al menos uno & 18.2 & 25.9 & 0.00 & 18.4 & 26.3 & 0.00 \\
\hline En ambos & 3.5 & 9.3 & 0.00 & 3.9 & 9.8 & 0.00
\end{tabular}

Pares que consumen drogas ilegales

\begin{tabular}{lrrrrrr} 
No & 95.0 & 79.1 & 0.00 & 90.9 & 69.4 & 0.00 \\
\hline Sí, pero no son amigos & 1.8 & 8.9 & 0.00 & 2.4 & 11.0 & 0.00 \\
\hline Sí, y son amigos & 3.2 & 11.9 & 0.00 & 6.7 & 19.7 & 0.00
\end{tabular}

No= Puede consumir alcohol pero no al punto de intoxicación (de "6 a II veces al año" hasta "nunca en los últimos doce meses, pero sí antes"

‡ Sí= Intoxicación al punto de tener dificultad para caminar o pararse (de "todos los días" hasta "más o menos una vez al mes")

$\S$ Ninguno de los padres consumió en ambas mediciones

\# Incluye los casos en que al menos uno de los padres consumió tabaco en la medición basal o en la segunda

mujeres estudiadas, se incrementó 6.4 puntos porcentuales la frecuencia del consumo de tabaco en el periodo de referencia ( 8.4 contra $14.4 \%$ ). Asimismo, durante el periodo de estudio se incrementó la prevalencia de consumo inmoderado de alcohol, antecedentes de consumo de drogas ilegales, inicio de vida sexual activa, entre otros factores más. En los 1455 hombres, la prevalencia del consumo de tabaco aumentó dos veces más en comparación con las mujeres, con un exceso de 11.5 puntos porcentuales en el periodo de estudio; al igual que las mujeres, se identificó un incremento porcentual de la exposición al consumo inmoderado de alcohol, drogas ilegales y vida sexual activa en relación con la mayor edad.

La población integrada por los estudiantes que persistieron en la conducta de fumar tabaco de manera activa, comparada con los estudiantes que mantenían la abstinencia (continuación en no consumir), fue de 2568 estudiantes; $66 \%$ corresponde a mujeres $(n=1695)$ y $34 \%(n=873)$ a hombres, con edades de 14 a 28 años (segunda medición) y un promedio de edad para ambos sexos de 16.9 años.

El cuadro II muestra las prevalencias de la continuidad del consumo de tabaco basadas en los 2568 estudiantes. Las prevalencias más altas de continuación del tabaquismo se registraron en los sujetos con bajo desempeño escolar, consumo inmoderado y frecuente de alcohol, antecedentes de consumo de drogas ilegales y síntomas concomitantes de depresión. En cuanto a la calificación autoinformada, aquellas mujeres que empeoraron o mantuvieron bajo rendimiento escolar acusan una persistencia en el consumo de tabaco de $7.2 \%$, mientras que entre los hombres la cifra es de $17.1 \%$. La prevalencia más elevada de exposición se identificó en hombres con antecedentes de consumo de drogas ilegales con $66.2 \%$ de persistencia en el consumo de cigarros. Asimismo, en la figura 2 se observa que existe una disminución de la diferencia proporcional de la continuidad del consumo de tabaco entre los grupos de alto y bajo rendimiento escolar conforme aumenta la edad.

La persistencia del consumo de tabaco entre los que iniciaron relaciones sexuales es más alta entre los hombres que entre las mujeres (36.9 y 20.6\%, respectivamente); cuando ambos padres consumieron tabaco en las dos mediciones, esta proporción fue de $11.6 \%$ en las mujeres y $23.8 \%$ en los hombres; por último, entre los incluidos en la categoría de síntomas depresivos, los porcentajes fueron de $23 \%$ en mujeres y $43.3 \%$ en hombres.

Respecto de la influencia de los pares, se observa que la persistencia del consumo de tabaco entre los que tenían amigos que consumían drogas ilegales es semejante para ambos sexos (66.7\% en mujeres y $67.7 \%$ en hombres), y el efecto de este hábito se refleja en mantener bajo o empeorar el desempeño escolar, en especial cuando se es amigo de un compañero de escuela que consume drogas ilegales, en ambos sexos.

En la figura 3 se advierte una mayor prevalencia de continuación del consumo de cigarrillos en función del 
Cuadro II

Distribución de PERsistencia del CONSUMO de TABACO EN ADOLESCENTES Y ADULTOS JÓVENES DEL ESTADO DE MORELOS DE LA POBLACIÓN DE ESTUdIO, I 998-200 I, ESTRATIFICADO POR SEXO ( $\mathrm{N}=2$ 568)

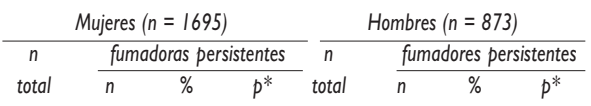

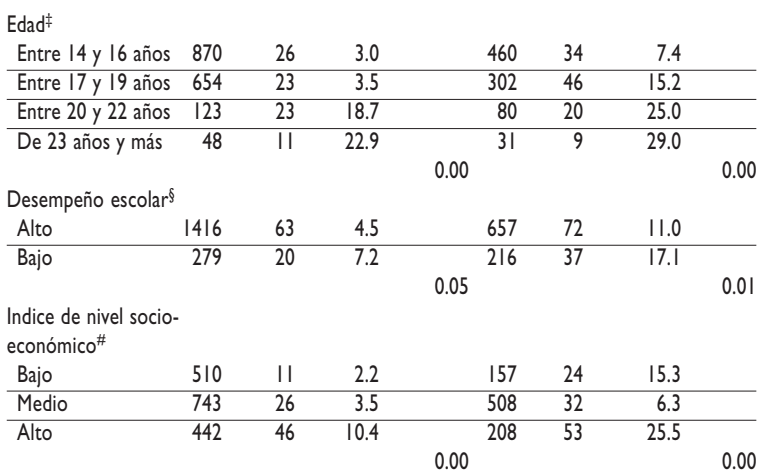

Lugar de residencia ${ }^{\S}$

\begin{tabular}{|c|c|c|c|c|c|c|}
\hline Rural & 39 & I & 2.6 & 13 & 2 & 15.4 \\
\hline Semiurbano & 398 & 15 & 3.8 & $|4|$ & 10 & 7.1 \\
\hline Urbano & 1258 & 67 & 5.3 & 719 & 97 & 13.5 \\
\hline
\end{tabular}

Consumo inmoderado

de alcoholई

$\begin{array}{lllllll}\text { Nunca } & 1208 & 10 & 0.8 & 537 & 9 & 1.7\end{array}$

\begin{tabular}{lrrrrrr}
\hline Ocasionalmente & 464 & 66 & 14.2 & 300 & 81 & 27.0 \\
\hline Frecuentemente & 23 & 7 & 30.4 & 36 & 19 & 52.8
\end{tabular}

Historia de consumo

de drogas ilegales $\S$

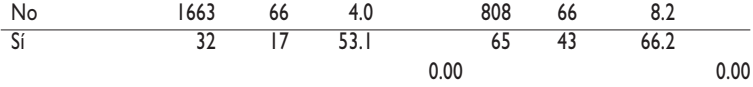

Antecedente de vida

sexual activa

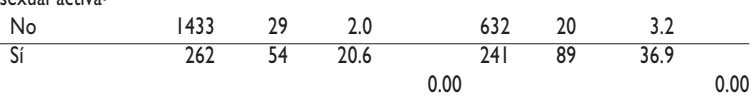

Percepción de

tabaquismo en padres $\$$

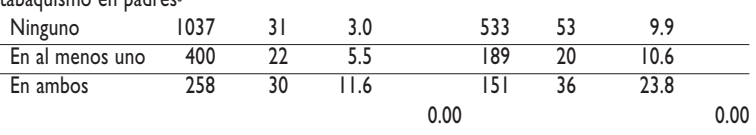

Antecedente de pares

que usan drogas

ilegales ${ }^{\S}$

\begin{tabular}{|c|c|c|c|c|c|c|}
\hline \multirow{2}{*}{\multicolumn{7}{|c|}{$\begin{array}{l}\text { No } \\
\text { Sí, pero no son }\end{array}$}} \\
\hline & & & & & & \\
\hline amigos & 295 & 42 & 14.2 & 216 & 48 & 22.2 \\
\hline Sí, y son amigos & 15 & 10 & 66.7 & 34 & 23 & 67.7 \\
\hline
\end{tabular}

Síntomas asociados

a depresión $\#, \&$

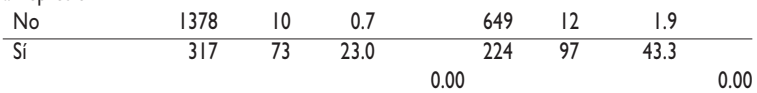

* Prueba de correlación $x^{2}$

₹ Segunda medición

$\S$ Ambas mediciones

\# Medición basal

\& Escala de Zung bajo rendimiento escolar en individuos con síntomas vinculados con depresión (48.6 contra $27.3 \%$ ), antecedente de vida sexual activa (44.2 contra $24.3 \%$ ) y consumo inmoderado de alcohol (36.2 contra 17.6\%). Por último, los estudiantes con nivel socioeconómico alto tuvieron una mayor prevalencia de tabaquismo y los estudiantes de bajo desempeño escolar (12.7\%) en comparación con los de alto desempeño escolar (7.2\%).

En el cuadro III se muestra el modelo de regresión logística múltiple, que incluyó los términos de interacción entre cada una de las variables y el sexo. Se presentan las $R M$ de la población total, estratificadas por género, así como el valor $p$ para el término de interacción. A partir de este modelo se observa que existe modificación de efecto por sexo sólo para las variables edad entre 20 y 22 años $(p=0.04)$, nivel socioeconómico medio $(p=0.04)$, nivel socioeconómico alto $(=0.02)$ y convivencia con amigos o pares que consumen drogas ilegales $(p=0.09)$. Esto significa que las mujeres con nivel socioeconómico alto tienen un riesgo mayor. Con base en esta información se decidió realizar un modelo de regresión logística para toda la población (sin estratificación por sexo) e incluir en él los términos de interacción que resultaron significativos al valor $p$. El cuadro IV presenta los factores vinculados con la persistencia del consumo de tabaco y desempeño escolar a través de un modelo de regresión logística múltiple, ajustado por sexo como covariable y otras variables, por ejemplo, nivel socioeconómico, estilo de vida, hábitos familiares e

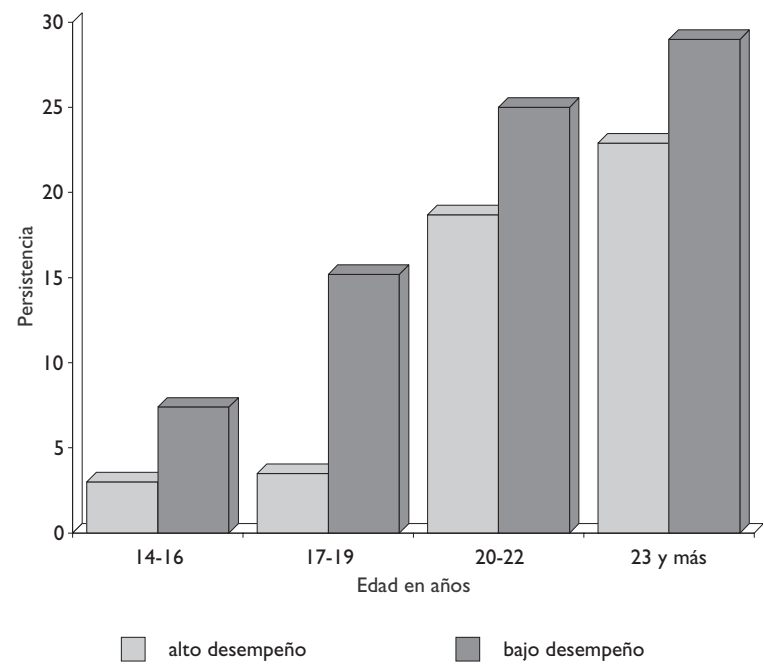

Figura 2. Persistencia del consumo de tabaco por edAd y función del desempeño escolar (Morelos, (998-200I) 


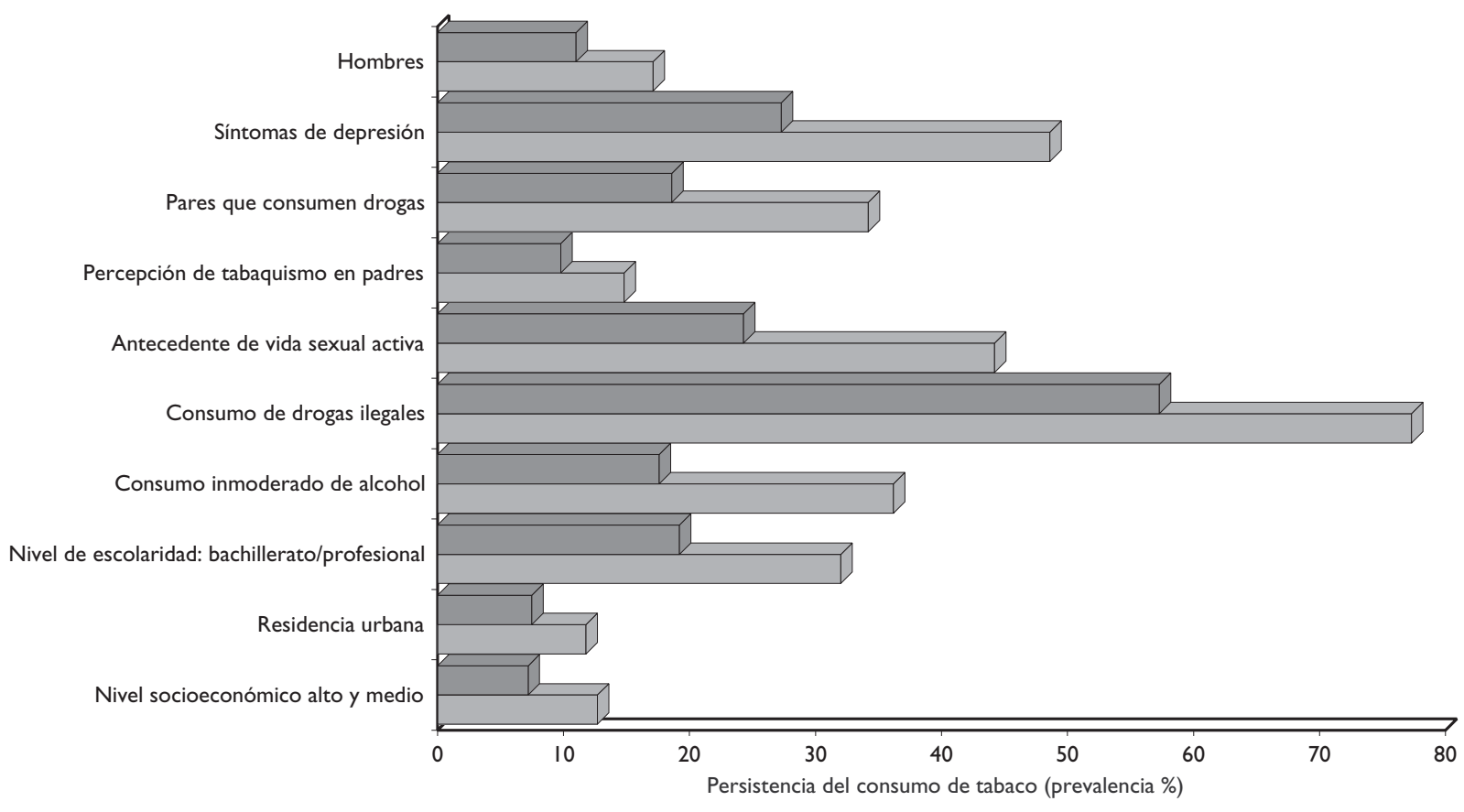

Alto desempeño escolar

Bajo desempeño escolar

Figura 3. Persistencia del consumo de tabaco de acuerdo con el desempeño escolar, las características SOCIOdEMOGRÁficas y PSICOLógICAS Y EL ESTILO de VIDA (MoRelos, 1998-200I)

influencia de pares, además de que se incluyeron los términos de interacción relacionados. De esta forma, en las mujeres de 20 a 22 años de edad se observa un riesgo 2.21 veces mayor de persistir en el consumo de tabaco (IC95\% 1.04-4.69), en tanto que para los hombres de la misma edad este nexo es protector en 67\% (IC95\% 0.14-0.75). Entre quienes mantuvieron bajo su desempeño escolar o lo empeoraron, las posibilidades de persistir en el consumo de tabaco fueron 2.48 veces mayores (IC95\% 1.51-4.07). En el índice de nivel socioeconómico se encontró una vinculación inversa para el sexo masculino, en los niveles medio y alto $(R M=0.33$; IC95\% $0.14-0.75 ; R M=0.28$; IC95\% 0.12-0.66, respectivamente). Entre la población existe un riesgo 4.22 veces mayor (IC95\% 2.36-7.52) de persistir en el consumo de tabaco en los estudiantes que consumen inmoderadamente alcohol en forma ocasional y de 7.93 veces en los que tienen esta exposición con frecuencia (IC95\% 3.14-19.99). El antecedente de consumo de drogas ilegales también se relacionó con la continuidad en el consumo de tabaco en 5.05 veces (IC95\% 2.61-9.77). El antecedente de relaciones sexuales incrementa 3.80 veces más las posibilidades de persistir en el hábito tabáquico (IC95\% 2.40-6.02). El tabaquismo en los padres y la continuación del consumo de tabaco resultó significativa para ambas categorías, pero de riesgo para aquellos que señalaron que ambos padres tenían este hábito $(R M=2.46$; IC95\% 1.51-4.02). El riesgo de proseguir en el consumo de tabaco entre las mujeres que conviven con amigos que recurren a drogas ilegales es 10.18 veces mayor (IC95\% 2.57-40.30). Por último, sufrir síntomas de depresión tiene una relación 9.88 veces mayor de persistir en el consumo de tabaco (IC95\% 5.74-17.02).

En el cuadro $\mathrm{V}$ se presentan los factores vinculados con la continuación del consumo de tabaco estratificados por elevado y bajo rendimiento escolar. Se observa que los factores relacionados con la persistencia del tabaquismo en función del desempeño escolar son similares en sujetos con bajo y elevado rendimiento escolar. Sin embargo, existe un exceso de riesgo en individuos con tendencia a disminuir su desempeño escolar en la mayoría de los factores estudiados, cuya diferencia es estadísticamente significativa. En este contexto, el consumo inmoderado de 
Cuadro III

Factores asociados a persistencia de consumo de tabaco en la población total de estudio Y estratificado por Sexo, en Adolescentes y adultos jóvenes de Morelos, 1998-200I ( $\mathrm{N}=2$ 568)

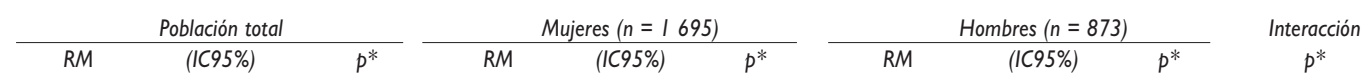

$\mathrm{Edad}^{\ddagger}$

\begin{tabular}{|c|c|c|c|c|c|c|c|c|c|c|}
\hline Entre 14 y 16 años & 1.00 & & & 1.00 & & & 1.00 & & & \\
\hline Entre 17 y 19 años & 1.09 & $0.67-1.77$ & 0.71 & 0.85 & $0.40-2.13$ & 0.68 & 1.49 & $0.67-3.35$ & 0.25 & 0.28 \\
\hline Entre 20 y 22 años & 1.29 & $0.72-2.31$ & 0.38 & 2.35 & $0.54-3.01$ & 0.04 & 0.69 & $0.63-3.43$ & 0.38 & 0.04 \\
\hline De 23 años y más & 0.95 & $0.44-2.07$ & 0.91 & 1.61 & $0.70-3.92$ & 0.41 & 0.57 & $0.25-1.25$ & 0.31 & 0.19 \\
\hline
\end{tabular}

Desempeño escolar $\$$

\begin{tabular}{|c|c|c|c|c|c|c|c|c|c|}
\hline Alto & 1.00 & & & 1.00 & & & & 1.00 & \\
\hline Bajo & 2.27 & $1.40-3.68$ & 0.00 & I.42-6.77 & 0.00 & 2.22 & I.13-4.17 & 0.01 & 0.53 \\
\hline
\end{tabular}

Indice de nivel socio-

económico\#

\begin{tabular}{|c|c|c|c|c|c|c|c|c|c|c|}
\hline Bajo & 1.00 & & & & 1.00 & & & & 1.00 & \\
\hline Medio & 0.71 & $0.40-1.25$ & 0.24 & 1.19 & $0.53-3.26$ & 0.70 & 0.33 & $0.14-0.75$ & 0.01 & 0.04 \\
\hline Alto & 0.72 & $0.40-1.31$ & 0.29 & 1.35 & $0.59-3.91$ & 0.52 & 0.31 & $0.13-0.75$ & 0.01 & 0.02 \\
\hline
\end{tabular}

Consumo inmoderado

de alcohol ${ }^{\S}$

\begin{tabular}{|c|c|c|c|c|c|c|c|c|c|c|}
\hline Nunca & 1.00 & & & & 1.00 & & & & 1.00 & \\
\hline Ocasionalmente & 4.29 & $2.42-7.59$ & 0.00 & 4.14 & $1.95-9.60$ & 0.00 & 4.14 & $1.74-10.13$ & 0.00 & 1.00 \\
\hline Frecuentemente & 7.06 & $2.79-17.85$ & 0.00 & 19.66 & $4.91-78.01$ & 0.00 & 5.32 & $1.60-19.66$ & 0.00 & 0.17 \\
\hline
\end{tabular}

Historia de consumo de

drogas ilegales ${ }^{\S}$

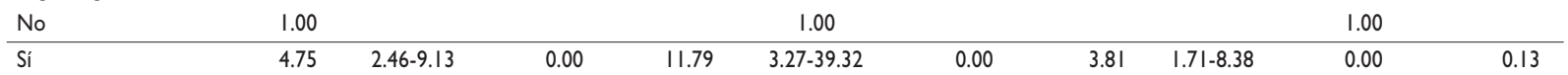

Antecedente de vida

sexual activa ${ }^{\S}$

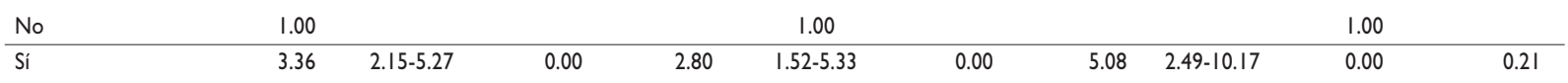

Percepción de tabaquismo

en padres $\S$

\begin{tabular}{|c|c|c|c|c|c|c|c|c|c|c|}
\hline Ninguno & 1.00 & & & & 1.00 & & & & 1.00 & \\
\hline En al menos uno & 1.60 & $0.95-2.69$ & 0.07 & 1.90 & $0.88-3.91$ & 0.09 & 1.39 & $0.61-2.90$ & 0.40 & 0.57 \\
\hline En ambos & 2.56 & $1.58-4.13$ & 0.00 & 3.19 & $1.49-6.19$ & 0.00 & 1.93 & $0.98-3.91$ & 0.06 & 0.32 \\
\hline
\end{tabular}

Antecedente de pares que

usan drogas ilegales ${ }^{\S}$

\begin{tabular}{|c|c|c|c|c|c|c|c|c|c|c|}
\hline No & 1.00 & & & & 1.00 & & & & 1.00 & \\
\hline Sí, pero no son amigos & 1.55 & $0.99-2.44$ & 0.05 & 1.89 & $0.97-3.62$ & 0.05 & 1.03 & $0.55-2.09$ & 0.91 & 0.21 \\
\hline Sí, y son amigos & 3.81 & $1.61-9.03$ & 0.00 & 10.70 & $2.45-41.84$ & 0.00 & 2.27 & $0.81-7.42$ & 0.14 & 0.09 \\
\hline
\end{tabular}

Síntomas asociados a

depresión $\#$ \&\&

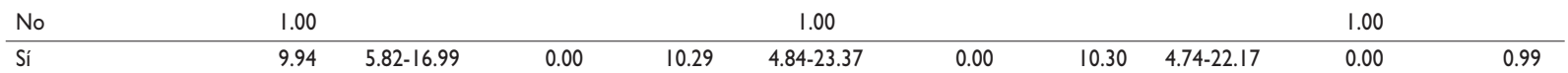

* Se considera $p$ estadísticamente significativa $(p<0.05)$

‡ Segunda medición

$\S$ Ambas mediciones

\# Medición basal

\&' Escala de Zung 


\section{Cuadro IV \\ FACTORES ASOCIADOS A PERSISTENCIA DE CONSUMO DE TABACO EN ADOLESCENTES Y ADULTOS JÓVENES $(\mathrm{N}=\mathbf{2 5 6 8})$}

\begin{tabular}{|c|c|c|c|}
\hline & \multicolumn{3}{|c|}{ Población total } \\
\hline & $R M$ & (IC95\%) & $p^{*}$ \\
\hline \multicolumn{4}{|l|}{$\mathrm{Edad}^{\ddagger}$} \\
\hline Entre 14 y 16 años & 1.00 & & \\
\hline Entre 17 y 19 años & 1.12 & $0.68-1.84$ & 0.63 \\
\hline \multicolumn{4}{|l|}{ Entre 20 y 22 años } \\
\hline Mujeres & 2.21 & $1.04-4.69$ & 0.03 \\
\hline Hombres & 0.33 & $0.14-0.75$ & 0.00 \\
\hline De 23 años y más & 0.93 & $0.42-2.02$ & 0.85 \\
\hline \multicolumn{4}{|l|}{ Desempeño escolar ${ }^{\S}$} \\
\hline Alto & 1.00 & & \\
\hline Bajo & 2.48 & $|.5|-4.07$ & 0.00 \\
\hline \multicolumn{4}{|c|}{ Índice de nivel socioeconómico\# } \\
\hline \multicolumn{4}{|l|}{ Mujeres } \\
\hline Bajo & 1.00 & & \\
\hline Medio & 1.29 & $0.54-3.09$ & 0.55 \\
\hline Alto & 1.61 & $0.68-3.79$ & 0.27 \\
\hline \multicolumn{4}{|l|}{ Hombres } \\
\hline Bajo & 1.00 & & \\
\hline Medio & 0.33 & $0.14-0.75$ & 0.00 \\
\hline Alto & 0.28 & $0.12-0.66$ & 0.00 \\
\hline
\end{tabular}

Consumo inmoderado de alcohol§

\begin{tabular}{lccc} 
Nunca & 1.00 & & \\
\hline Ocasionalmente & 4.22 & $2.36-7.52$ & 0.00 \\
\hline Frecuentemente & 7.93 & $3.14-19.99$ & 0.00
\end{tabular}

Historia de consumo de drogas ilegales $\S$ No $\quad 1.00$

\begin{tabular}{llll}
\hline Sí & 5.05 & $2.61-9.77$ & 0.00
\end{tabular}

Antecedente de vida sexual activa ${ }^{\S}$

\begin{tabular}{llll} 
No & 1.00 & & \\
\hline Sí & 3.80 & $2.40-6.02$ & 0.00
\end{tabular}

Percepción de tabaquismo en padres ${ }^{\S}$

\begin{tabular}{llll} 
Ninguno & 1.00 & & \\
\hline En al menos uno & 1.62 & $0.96-2.74$ & 0.07 \\
\hline En ambos & 2.46 & $1.5 I-4.02$ & 0.00
\end{tabular}

Antecedente de pares que usan drogas ilegales $\S$

No $\quad 1.00$

\begin{tabular}{llll}
\hline Sí, pero no son amigos & 1.42 & $0.89-2.25$ & 0.13
\end{tabular}

Sí, y son amigos

$\begin{array}{llll}\text { Mujeres } & 10.18 & 2.57-40.30 & 0.00\end{array}$

$\begin{array}{llll}\text { Hombres } & 2.33 & 0.80-6.72 & 0.11\end{array}$

Síntomatología asociada a depresión $\#, \&$ $\begin{array}{ll}\text { No } & 1.00\end{array}$

\begin{tabular}{llll}
\hline Sí & 9.88 & $5.74-17.02$ & 0.00
\end{tabular}

* Se considera $p$ estadísticamente significativa $(p<0.05)$

* Segunda medición

$\S$ Ambas mediciones

\# Medición basal

\& Escala de Zung alcohol tiene un exceso de riesgo $(18.6, p<0.01)$ en personas con bajo desempeño escolar; este efecto se reconoce también en sujetos con antecedentes de consumo de drogas ilegales (exceso de riesgo 19.9, $p>0.05$ ), aunque la diferencia no fue estadísticamente significativa. Asimismo, el antecedente de inicio de vida sexual activa se correlaciona de manera sólida con la edad (coeficiente de correlación de Pearson $=0.2513 ; p=0.000$ ) y tuvo un exceso de riesgo de $19.9(p<0.01)$; el antecedente de pares en la escuela que consumen drogas ilegales tuvo un exceso de riesgo en sujetos con bajo rendimiento escolar de $15.5(p<0.01)$ en comparación con los de alto desempeño escolar y, por último, los estudiantes con síntomas secundarios a la depresión tuvieron un elevado exceso de riesgo $(21.3, p<0.001)$ cuando se compararon deficiente y elevado rendimiento escolar.

\section{Discusión}

Los sujetos con bajo desempeño escolar son un grupo vulnerable de exposición a conductas no saludables, como lo revela este estudio. En realidad, esta cohorte de estudiantes del estado de Morelos no pretende mostrar un nexo causal entre el bajo desempeño escolar y el tabaquismo, sino establecer la estrecha correlación del rendimiento escolar bajo y las múltiples conductas de alto riesgo, entre las que se incluyen las siguientes: elevada prevalencia en la continuación del tabaquismo, consumo inmoderado de alcohol y consumo de drogas ilegales. Asimismo, los individuos con bajo desempeño escolar presentan una mayor prevalencia de síntomas relacionados con la depresión, así como mayor frecuencia de vida sexual activa, entre otros. En contraposición, los estudiantes con mejor rendimiento académico son más propensos a elegir conductas saludables, tal y como ya se ha descrito. ${ }^{18}$

Un deficiente desempeño escolar se vincula con la persistencia de tabaquismo al margen de la presencia de otros cofactores y provee un exceso de riesgo. Se ha determinado que existe relación entre los bajos logros académicos durante la adolescencia y la presencia del hábito, aunque se considera que los fumadores casi nunca se diferencian de los no fumadores en términos del coeficiente intelectual. ${ }^{19}$ Para este estudio se utilizó un indicador de nivel educativo adquirido utilizado en la mayoría de los países: las calificaciones escolares. Este indicador es básico, ya que refleja las expectativas del alumno, los docentes y la propia familia acerca de lo que es el logro y el éxito escolar.

Diversos estudios que han intentado caracterizar los estilos de vida saludables en adolescentes han incluido entre otros el "elevado desempeño académico"; estos estudios han enfatizado la importancia de la escuela, 


\section{Cuadro V

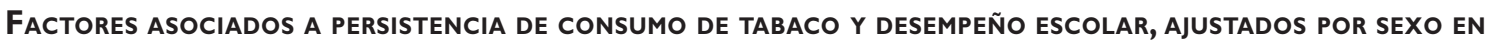 ADOLESCENTES Y ADULTOS JÓVENES $(\mathrm{N}=2568)$}

\begin{tabular}{|c|c|c|c|c|}
\hline \multicolumn{4}{|c|}{ Desempeño escolar } & \multirow[b]{3}{*}{$\begin{array}{l}\text { Diferencia de } \\
\text { prevalencias }\end{array}$} \\
\hline & mpeño escolar & & ipeño escolar & \\
\hline $\begin{array}{c}\text { Prev } \\
\%\end{array}$ & $\begin{array}{c}(n=495) \\
R M(I C 95 \%)\end{array}$ & $\begin{array}{c}\text { Prev } \\
\%\end{array}$ & $\begin{array}{l}(n=2073) \\
R M(I C 95 \%)\end{array}$ & \\
\hline
\end{tabular}

Edad ${ }^{\ddagger}$

\begin{tabular}{lrrrrrr} 
Entre 14 y 16 años & 6.0 & 1.00 & 4.1 & 1.00 & 1.9 & 0.21 \\
\hline Entre 17 y 19 años & 14.7 & $4.30(1.27-14.49)$ & 5.3 & $0.77(0.44-1.33)$ & 9.3 & 0.00 \\
\hline Entre 20 y 22 años & 37.0 & $0.96(0.23-4.03)$ & 18.8 & $1.41(0.75-2.64)$ & 18.3 & 0.09 \\
\hline De 23 años y más & 30.0 & $0.46(0.07-2.95)$ & 24.6 & $1.16(0.51-2.63)$ & 5.4 & 0.78
\end{tabular}

Sexo

\begin{tabular}{lrrrrrr} 
Mujeres & 7.2 & 1.00 & 4.5 & 1.00 & 2.7 & 0.06 \\
\hline Hombres & 17.1 & $1.29(0.85-1.95)$ & 11.0 & $2.75(1.53-4.93)$ & 6.2 & 0.03
\end{tabular}

Indice de nivel socioeconómico $\$$

\begin{tabular}{lrcrcrr} 
Bajo & 8.3 & 1.00 & 4.5 & 1.00 & 3.8 & 0.10 \\
\hline Medio y alto & 12.7 & $2.00(0.58-6.78)$ & 7.2 & $0.63(0.34-1.15)$ & 5.5 & 0.00
\end{tabular}

Lugar de residencia

\begin{tabular}{lcccccc} 
Rural & 10.7 & 1.00 & 3.2 & 1.00 & 7.5 & 0.00 \\
\hline Urbano & 11.8 & $0.95(0.33-2.75)$ & 7.5 & $1.85(0.95-3.60)$ & 4.3 & 0.01
\end{tabular}

Escolaridad

\begin{tabular}{lrrrrrr} 
Secundaria & 9.2 & 1.00 & 4.5 & 1.00 & 4.7 & 0.00 \\
\hline Bachillerato y profesional & 32.0 & $0.53(0.17-1.62)$ & 19.2 & $1.20(0.73-1.97)$ & 12.8 & 0.11
\end{tabular}

Consumo inmoderado de alcohol ${ }^{\#}$

\begin{tabular}{lrrrrrr} 
No & 0.5 & 1.00 & 1.2 & 1.00 & -0.6 & 0.31 \\
\hline Sí & 36.2 & $21.37(4.32-105.65)$ & 17.6 & $3.28(1.76-6.09)$ & 18.6 & 0.00
\end{tabular}

Historia de consumo de drogas ilegales $\$$

\begin{tabular}{lrrrrrr} 
No & 8.5 & 1.00 & 4.6 & 1.00 & 3.6 & 0.00 \\
\hline Sí & 77.3 & $13.79(1.90-99.97)$ & 57.3 & $4.67(2.32-9.42)$ & 19.9 & 0.42
\end{tabular}

Antecedente de inicio de vida sexual activa ${ }^{\#}$

\begin{tabular}{lrrrrrr} 
No & 2.8 & 1.00 & 2.3 & 1.00 & 0.5 & 0.53 \\
\hline Sí & 44.2 & $5.7(1.99-16.3 I)$ & 24.3 & $3.04(1.85-5.01)$ & 19.9 & 0.00
\end{tabular}

Percepción de tabaquismo en padres\#

\begin{tabular}{lrccccc} 
Ninguno & 9.1 & 1.00 & 4.5 & 1.00 & 4.5 & 0.00 \\
\hline En al menos uno & 14.8 & $2.24(0.84-5.95)$ & 9.8 & $2.15(1.36-3.40)$ & 5.1 & 0.06
\end{tabular}

Antecedente de pares que usan drogas ilegales $\#$

\begin{tabular}{lrrrrrr} 
No & 4.3 & 1.00 & 3.2 & 1.00 & 1.0 & 0.34 \\
\hline Sí & 34.2 & $1.47(0.51-4.22)$ & 18.6 & $1.82(1.12-2.96)$ & 15.5 & 0.00
\end{tabular}

Síntomas asociados a depresión

\begin{tabular}{lrrrrrr} 
No & 1.5 & 1.00 & 0.9 & 1.00 & 0.6 & 0.34 \\
\hline Sí & 48.6 & $18.37(5.49-61.4 I)$ & 27.3 & $10.55(5.70-19.52)$ & 21.3 & 0.00
\end{tabular}

* Prueba exacta de Fisher para diferencia de prevalencias

¥ Segunda medición

$\S$ Medición basal

\# Ambas mediciones

\& Escala de Zung

* Modelo de regresión logística múltiple con término de interacción por sexo 
los pares y el apoyo de lo padres en las conductas saludables. ${ }^{18}$

Por otra parte, de la percepción general de los alumnos y profesores se advierte que los estudiantes con un mejor desempeño escolar no fuman, en comparación con quienes poseen desempeños bajos, ${ }^{20}$ influidos por los estilos de vida no saludables a los que se exponen de modo concomitante. A este respecto, se ha documentado que los fumadores obtienen en promedio calificaciones menores que los no fumadores. ${ }^{19}$ En los ámbitos social y psicológico se ha identificado una relación entre las expectativas educacionales de los adolescentes y su relación con el tabaquismo: los adolescentes que fuman se ven a sí mismos, y a otros que también fuman, como menos exitosos desde el punto de vista académi$\mathrm{co}$, y en contraposición ven a los no fumadores como más exitosos en su rendimiento escolar. ${ }^{21,22}$

Resultados similares a los de este trabajo se han descritos en protocolos anteriores en los que se observa que el tabaquismo a edades tempranas predice dicho hábito en edades posteriores. ${ }^{23}$ En este estudio se halló que la persistencia en el consumo de tabaco es directamente proporcional a la edad pero, a diferencia de otros estudios, ${ }^{24,25}$ no existe diferencia por género. A pesar de que se ha documentado que las mujeres han incrementado la frecuencia de consumo de tabaco, existe un consenso sobre su tendencia a adoptar decisiones que benefician su salud en comparación con sus contrapartes varones. ${ }^{18}$

Los principales determinantes de la persistencia de tabaquismo son el consumo inmoderado de alcohol y drogas ilegales, ${ }^{26-28}$ pero estas variables de exposición son más prevalentes en sujetos con bajo desempeño escolar. ${ }^{9,13,29}$ En este estudio se encontró un exceso de riesgo al evaluar las diferencias de prevalencias de adicciones en personas con bajo rendimiento escolar. En este contexto se ha notificado el abuso del alcohol como una de las causas de inasistencia a la escuela y su dependencia se vincula con un patrón psíquico de inadaptación social y, por lo tanto, escolar. En el caso de un alto consumo de alcohol, se reduce la posibilidad de llegar a obtener el grado universitario. ${ }^{30}$ De igual modo, se ha examinado el efecto de la exposición a la marihuana y el consumo inmoderado de alcohol en el desempeño escolar y se han encontrado efectos adversos significativos, sea en la tasa de deserción escolar ${ }^{9} \mathrm{o}$ la tasa de obtención del grado de secundaria. ${ }^{14}$ Esto suministra una posible relación entre el incremento del consumo de tabaco y el bajo desempeño escolar, determinada por variables relacionadas con estilos de vida. ${ }^{15}$

De modo consistente con las teorías del aprendizaje social (como la teoría cognitiva social de Bandura), los niños aprenden conductas al copiarlas de los padres y éstos fungen como modelos. El consumo de tabaco en los padres se considera un predictor significativo para la persistencia del tabaquismo en los adolescentes, sin importar la presencia de otros factores de riesgo. ${ }^{31} \mathrm{En}$ los estudiantes de Morelos se encontró una relación directamente proporcional de proseguir el consumo de tabaco cuando ambos padres mantienen este hábito. Además, las condiciones del ámbito escolar-como la presencia de tabaquismo en los profesores-y la influencia de la conducta tabáquica de otros miembros de la familia elevan el riesgo de persistencia. ${ }^{5,32}$ En estudios de mujeres con madres fumadoras se ha señalado que tienen menos logros académicos en comparación con los hijos de las de madres no fumadoras. ${ }^{33}$

Respecto de la vida sexual activa, se ha documentado una prevalencia mayor de tabaquismo en adolescentes que inician las relaciones sexuales en fase temprana. En general, son los varones quienes inician a edades más tempranas su actividad sexual. ${ }^{18}$ Cabe mencionar que la ocurrencia de múltiples conductas de riesgo se incrementa con la edad, dado que la mayoría de los adolescentes adquiere conductas no saludables de forma gradual, más que de manera simultánea. ${ }^{34}$

Los síntomas depresivos y la ansiedad han resultado predictores del inicio de fumar en adolescentes en múltiples estudios; en fumadores, predicen la persistencia del consumo de tabaco, tal y como se muestra en los estudiantes de Morelos; el efecto más sólido se identifica en personas con tendencia a mantener bajo el desempeño escolar o disminuirlo a lo largo del tiempo. Los estudios sugieren que la depresión, más que consecuencia del tabaquismo, puede influir en la consolidación del consumo de tabaco en el adolescente. ${ }^{35}$ De manera adicional, un adolescente que presenta síntomas depresivos puede ser más susceptible a la influencia de sus pares para iniciarse en la experimentación y adherencia al consumo. ${ }^{36}$ En los estudiantes de Morelos con bajo rendimiento escolar se observa una mayor prevalencia de síntomas depresivos y exceso del riesgo de tabaquismo.

$\mathrm{Al}$ analizar el efecto de la escolaridad y su nexo con el hábito de fumar se ha observado que el riesgo de adquirir el hábito se relaciona en grado significativo con el ambiente de los pares y que esta vinculación se incrementa conforme el adolescente accede a otro nivel de enseñanza. ${ }^{37}$ De igual manera, se ha encontrado que la relación mayor en los distintos grados escolares tiene lugar en los niveles académicos más altos, en los cuales los estudiantes reconocieron tener algún amigo consumidor de tabaco. ${ }^{38}$ Esta vinculación entre la persistencia del consumo influida por los pares puede explicarse desde la perspectiva de la teoría de los problemas del comportamiento. ${ }^{39}$ En Morelos, el antecedente de compañeros que consumen drogas fue más 
notorio en sujetos con pobre desempeño escolar y un sólido predictor de continuación del tabaquismo.

Respecto de las limitaciones potenciales del estudio, una es la fuente de información, dado que proviene de encuestas autoaplicadas que tal vez no correspondan a la frecuencia real de consumo de tabaco en la población en estudio; empero, los trabajos previos han documentado una gran correlación entre el informe por cuestionarios autoaplicables y biomarcadores de consumo de tabaco, con límites estrechos de clasificación errónea. ${ }^{40}$ Aun con esta potencial limitación, los resultados encontrados concuerdan con otros desarrollados en el contexto educativo. En este sentido, los resultados son comparables porque consideran la posible relación entre la persistencia del consumo de tabaco y el cambio del desempeño académico; además, en todos los casos el grupo de referencia es el de mayor rendimiento escolar, en comparación con quienes muestran un menor desempeño académico. ${ }^{32}$

$\mathrm{Al}$ tratarse de una cohorte de estudiantes, uno de los criterios de inclusión fue la inscripción en la escuela, lo que redujo la representatividad de la población de estudio respecto de la población original, ya que los estudiantes que se mantienen tendrían prevalencias menores de los factores de riesgo; por lo tanto, se puede presuponer que los resultados pueden estar subestimados. La posibilidad de un sesgo de selección se considera poco probable, dado que no hubo cambios en el sentido de la distribución de la población de estudio de acuerdo con las categorías de exposición entre la población de la medición basal en relación con la segunda medición. En cuanto al sesgo de información, el estudio se basó en el autoinforme de los participantes y la vinculación podría estar subestimada (aunque la declaración del hábito se considera una estimación válida de la conducta real) ${ }^{38,41}$ por esto se asume que el error se distribuye aleatoriamente entre los grupos expuestos y no expuestos y cualquier factor puede influir de modo no diferencial en cada uno de ellos. Los potenciales factores de confusión se han controlado mediante la inclusión de variables relacionadas con la persistencia del hábito y estilos de vida en cada uno de los modelos probabilísticos múltiples. Se puede afirmar que el nexo entre bajo desempeño escolar y persistencia del consumo de tabaco es estrecha y se mantiene al ajustar por el resto de las variables.

Se puede concluir que los resultados obtenidos son un reflejo del incremento del consumo de tabaco que se ha observado en los últimos 20 años en la población de adolescentes en México. ${ }^{42,43}$ Los registros de esta adicción muestran que en este país el grupo más vulnerable de inicio de exposición lo constituyen los estudiantes del nivel secundaria, en el que se encuentra la frecuen- cia más elevada de inicio de consumo de tabaco $(40.7 \%) ;{ }^{44}$ a este respecto, en los últimos 20 años la proporción de adolescentes que se inicia en el consumo de tabaco a más temprana edad aumentó de tal forma que uno de cada 10 adolescentes hombres de áreas urbanas y rurales empezó a fumar antes de los 11 años de edad. ${ }^{45}$

Asimismo, de acuerdo con la Encuesta sobre Tabaquismo en Jóvenes que suministra información sobre adolescentes de secundaria de 12 a 15 años de edad, en México la mitad de los estudiantes (51.1\%) ha experimentado o probado el cigarro alguna vez, o bien ha tenido contacto con algún producto del tabaco, y la brecha de prevalencia por género ha desaparecido (19.9\% en hombres y $19.1 \%$ en mujeres). ${ }^{46}$

Por último, en la población de estudiantes mexicanos, hoy en día se cuenta con un cuerpo de conocimientos que se ha enfocado en identificar los factores que incrementan o disminuyen la presencia de comportamientos saludables. ${ }^{8,28}$ En este contexto, se conoce que la principal influencia en la continuación del hábito es la exposición a otro tipo de adicciones (consumo inmoderado de alcohol y drogas ilegales) y que en el plano escolar los individuos con bajo desempeño académico son sujetos más vulnerables a estilos de vida no saludables. La siguiente etapa dentro de la política pública contra el tabaquismo debe ser el desarrollo y la aplicación de programas de intervención educativa en población adolescente que instituyan diversas medidas, con énfasis en grupos vulnerables de alto riesgo, como son los estudiantes con rendimiento escolar deficiente.

De manera adicional, las instituciones educativas deben asumir su responsabilidad social como instancias que promueven comportamientos que influyen en la salud integral del individuo e implantar programas que propicien actividades extracurriculares e impulsen la actividad física. También debe promoverse el asesoramiento y el consejo a estudiantes, además de activar programas que anulen los efectos adversos de la adopción de este hábito, el consumo persistente y, por supuesto, sus consecuencias en el éxito escolar de la población estudiantil.

\section{Agradecimientos}

Este proyecto de investigación fue financiado por el Consejo Nacional de Ciencia y Tecnología de México, Salud.2003-C01-59 y Salud. 2003-C01-78.

\section{Referencias}

I. Castillo I, Balaguer I, Duda J. Las teorías personales sobre el logro académico y su relación con la alienación escolar. Psicothema 2003:75-8I. 
2. La teoría de la acción razonada de Martín Fishbein. Disponible en: http://www.filos.unam.mx/POSGRADO/seminarios.

3. Diccionario Porrúa de la Lengua Española. México: Porrúa, 1977. 4. Jackson KM, Sher KJ, Cooper ML, et al. Adolescent alcohol and tobacco use: onset, persistence and trajectories of use across two samples. Addiction 2002;97:5I7-53I.

5. Johnson EO, Chase GA, Breslau N. Persistence of cigarette smoking: familial liability and the role of nicotine dependence. Addiction 2002;97(8): 1063-1070.

6. Begg DJ, Langley JD, Stephenson S. Identifying factors that predict persistent driving alter drinking, unsafe driving alter drinking, and driving alter using cannabis among young adults. Accid Anal Prev 2003;35:669-675

7. Flay BR, Hu FB, Richardson J. Psychosocial predictors of different stages of cigarette smoking among high school students. Prev Med 1998;27:A9-AI8.

8. Arillo-Santillán E, Fernández E, Hernández-Ávila M et al. Prevalencia de tabaquismo y bajo desempeño escolar en estudiantes de II a 24 años de edad del Estado de Morelos, México. Salud Publica Mex 2002;44(supl l):S54-S66.

9. Bray J, Zarkin G, Ringwalt C, et al. The relationship between marijuana initiation and dropping out of high school. Health Econ 2000;9:9-18.

10. Tyas SL, Pederson LL. Psychosocial factors related to adolescent smoking: a critical review of the literature. Tob Control 1998;7:409-420. II. Navarro R. El rendimiento académico: concepto, investigación y desarrollo. http:www.redcientifica.com/doc/doc20030623060l.html 12. Wetzels J], Kremers SP,Vitória PD, et al.The alcohol-tobacco relationship: a prospective study among adolescents in six European countries. Addiction 2003;98(I2):1755-1763.

13. Conrad KM, Flay BR, Hill D. Why children start smoking cigarettes: predictors of onset. Br J Addict 1992;87: I7 I I-1724.

14. Simons-Morton B, Haynie DL, Crump AD, et al. Peer and parent influences on smoking and drinking among early adolescents. Health Educ Behav 200I Feb;28(I):95-107.

15. Jefferis B, Graham H, Manor O, Power C. Cigarette consumption and socio-economic circumstances in adolescence as predictors of adult smoking. Addiction 2003;98(I2): I765-1772.

16. Lazcano-Ponce E, Hernández B, Cruz-Valdez A, et al. Chronic disease risk factors among healthy adolescents attending public schools in the State of Morelos, Mexico. Arch Med Res 2003;34:222-236.

17. Waldrom I. Patterns and causes of gender differences in smoking. Soc Sci Med 1991;32:989-1005.

18. Kramer E. Defining and understanding healthy lifestyles choices for adolescents. Int J Adolesc Med Health 2004;35:26-33.

19. Matarazzo JD, Saslow G. Psychological and related characteristics of smokers and nonsmokers. Psychol Bull 1960;57:493-5।3.

20. Bewley BR, Bland JM. Academic performance and social factors related to cigarette smoking by schoolchildren. Br J Prev Soc Med 1977(31);18-24.

21. Creswell WH, Huffman WJ, Stone DB. Youth smoking behavior characteristics and their educational implications. A report of the University of Illinois antismoking education study. Champaign: University of Illinois, 1970.

22. Laoye JA, Creswell WH, Stone DB. A cohort study of I 205 secondary school smokers. J Sch Health 1972;42:47-52.

23. Friestad C, Klepp KI. From experimentation to habitual smoking. A three-year follow-up study of smoking behavior of adolescents. Tidsskr Nor Laegeforen 1996;20:1 I6(5):635-638.
24. Gritz ER. Cigarette smoking in a multiethnic population of youth: methods and baseline findings. Prev Med 1998;27:365-384.

25. Azevedo A, Machado AP, Barros H. Tobacco smoking among portuguese high-school students. Bull World Health Organ 1999;77(6):509-514.

26. Goodstadt MS, Chan GC, Sheppard MA. Developmental and generational trends in alcohol, cannabis and tobacco use- a ten year cohort analysis. Drug Alcohol Depend 1982;10(4):303-320.

27. Degenhardt L, Lynskey M, Hall W. Cohort trends in the age of initiation of drug use in Australia. Aust N Z J Public Health 2000;24(4):42I-426.

28. Arillo-Santillan E, Lazcano-Ponce E, Hernandez-Avila M, et al. Associations between individual and contextual factors and smoking in I3 293 mexican students. Am J Prev Med 2005;28(I):41-5I.

29. Lynskey M, Hall W. The effects adolescent cannabis use on educational attainment: a review. Addiction 2000;95(II):162I-1630. 30. Cook PJ, Moore MJ. Drinking and schooling.J Health Econ 1993; | 2:4II-429.

3I. Johnson CC, Li D, Perry CL, et al. Fifth through eighth grade longitudinal predictors of tobacco use among a racially diverse cohort: CATCH.J Sch Health 2002;72(2):58-64.

32. Coogan PF,Adams M, Geller AC, et al. Factors associated with smoking among children and adolescents in Connecticut. Am J Prev Med 1998; 15(I):17-24.

33. Hover SJ, Gaffney LR. Factors associated with smoking behavior in adolescent girls. Addict Behav 1988; I3(2): I39- I45.

34. Kulbok P, Cox Ch. Dimensions of adolescent health behavior.J Adolesc Health 2002; I:394-400.

35. Patton GC. Is smoking associated with depresión and anxiety in teenagers? Am J Public Health 1996;86:225-300.

36. Patton GC. Depresion, anxiety, and smoking initiation: a prospective study over 3 years. Am J Public Health 1998;88.

37. Alexander C, Piazza M, Mekos D, et al. Peers, schools, and adolescent cigarette smoking. J Adolesc Health 200I;29(I):22-30.

38. Mendis S. Tobacco use in a cohort of children in Sri Lanka. $\mathrm{Br} J$ Addict 1990;85(3):397-398.

39. Jessor R. Problem-behavior theory, psychosocial development, and adolescent problem drinking. Br J Addict 1987;82:331-342.

40. Wells AJ, English PB, Posner SF, et al. Misclassification rates for current smokers misclassified as nonsmokers. Am J Public Health 1998;88:1503-1593.

4I. Morabia A, Bernstein M, Curtin F, et al.Validation of self-reported smoking status by simultaneous measurement of carbon monoxide and salivary thiyanate. Prev Med 2001;32:82-88.

42. Secretaría de Salud. Encuesta Nacional de Adicciones 1988. México, DF: SSA, 1990.

43. Secretaría de Salud. Encuesta Nacional de Adicciones 1998. México, DF: SSA, 1999.

44. Secretaría de Salud. Encuesta Nacional de Adicciones 2002. México, DF: Secretaría de Salud, 2002.

45. Secretaría de Salud. El consumo de tabaco en México y Encuesta Nacional de Adicciones, 1998 (Tabaco). México.

46. Valdés-Salgado R, Meneses-González F, Lazcano-Ponce EC, et al. Encuesta sobre Tabaquismo en Jóvenes, México 2003. Cuernavaca, Morelos: Instituto Nacional de Salud Pública, 2004. 